\title{
Development of a New In Vivo Optical Probe for Biological Diagnosis and Therapy
}

\author{
Michitaka Ozaki, Takeaki Ozawa, and Yuma Yamada
}

\begin{abstract}
Real-time imaging of the specific markers of lesions in the living body will provide valuable information in various physiopathological situations. Clinically, real-time imaging will definitely aid accurate diagnosis and rational therapy, especially in the surgical field.

In this chapter, we describe some unique optical probes for "biological imaging" and our recent challenge in undertaking development of a new type of in vivo probe. The reduction-oxidation-sensitive green fluorescent protein (roGFP) and bioluminescent luciferase probe for caspase-3 activity have been useful for understanding of the dynamic changes of liver redox states and apoptotic cell death. To overcome the difficulty of imaging in deeper lesions by optical probes, we newly developed a far-red bioluminescent probe. Lastly, we have undertaken the challenge to develop an innovative optical probe that switches "on" only when the probe recognizes a target molecule to reduce non-specific signals in vivo. The project of developing this unique probe is still underway.

Regarding a carrying system of the probe into cells in vivo, we have developed a liposome with cell-penetrating octa-arginine peptides (R8) and a pH-sensitive fusogenic peptide (GALA), which delivers the functional proteins into cells efficiently and rapidly in vivo.

We believe that these optical probes will provide a new avenue toward new diagnosis and therapy to come in the future.
\end{abstract}

Keywords Optical probe $\bullet$ In vivo imaging • Fluorescence • Bioluminescence • Oxidative stress $\bullet$ Apoptosis $\bullet$ Liver $\bullet$ Liposome

\footnotetext{
M. Ozaki, M.D., Ph.D. (四)

Laboratory of Molecular and Functional Bio-Imaging (LMFBI), Faculty of Health Sciences, Graduate School of Health Sciences, Hokkaido University,

N-12, W-5, Kita-ku, Sapporo, Hokkaido 060-0812, Japan

e-mail: ozaki-m@med.hokudai.ac.jp

T. Ozawa

Department of Chemistry, School of Science, The University of Tokyo,

7-3-1 Hongo, Bunkyo-ku, Tokyo 113-0033, Japan

Y. Yamada

Laboratory for Molecular Design of Pharmaceutics, Faculty of Pharmaceutical Sciences,

Hokkaido University, N-12, W-5, Kita-ku, Sapporo, Hokkaido 060-0812, Japan
}

K. Nakao et al. (eds.), Innovative Medicine, DOI 10.1007/978-4-431-55651-0_22 


\section{Abbreviations}

$\begin{array}{ll}\text { AdpcFluc } & \text { Adenovirus vector encoding pcFluc-DEVD } \\ \text { Dlk1 } & \text { Human Delta-like protein } \\ \text { AP-1 } & \text { Activator protein 1 } \\ \text { ATP } & \text { Adenosine triphosphate } \\ \text { BG } & \text { Benzylguanine } \\ \text { BRET } & \text { Bioluminescence resonance energy transfer } \\ \text { CLuc } & \text { Cypridina luciferase } \\ \text { DEVD } & \text { Asp-Glu-Val-Asp (a substrate sequence of caspase-3) } \\ \text { FBP } & \text { Far-red bioluminescent protein } \\ \text { FLuc } & \text { Firefly luciferase } \\ \text { FlucC-SNAP } & \text { C-terminal fragment of luciferase conjugated with SNAP } \\ \text { FlucN-SNAP } & \text { N-terminal fragment of luciferase conjugated with SNAP } \\ \text { H/R } & \text { Hypoxia and reoxygenation } \\ \text { I/R } & \text { Ischemia and reperfusion } \\ \text { MAPK } & \text { Mitogen-activated Protein Kinase } \\ \text { MEND } & \text { Multi-functional envelope-type nano device } \\ \text { NF-kB } & \text { Nuclear factor-kappa B } \\ \text { OS } & \text { Oxidative stress } \\ \text { pcFluc-DEVD } & \text { Cyclic luciferase reflecting caspase-3 activity } \\ \text { PET } & \text { Positron emission tomography } \\ \text { roGFP } & \text { Reduction-oxidation sensitive green fluorescent protein } \\ \text { ROS } & \text { Reactive oxygen species } \\ \text { R8 } & \text { Octa-arginine peptide } \\ \text { SNAP } & \text { A } 182 \text { resides }\end{array}$

SNAP A 182 residues polypeptide that can be fused to any protein of interest

\section{Introduction}

Real-time monitoring of molecular markers of lesions in the living body will provide valuable information on various physiopathological phenomena. This will aid understanding of lesions qualitatively as well as quantitatively. Clinically, this will lead to more accurate diagnosis and rational therapy. Especially in the surgical field, this kind of technology will enable us to "see" the biological characteristics (and potential pathological/progressive behavior) of the lesions during operation as well as its location, size, and expansion, which will definitely help the surgeon select the best surgical procedure for the patient.

The conventional imaging technologies commonly used in clinical practice include CT, PET-CT, and MRI. These technologies provide useful and geographical information about the location, size, shape, and expansion (including distant metastasis), and aid accurate clinical diagnosis and pre-operative staging. However, they 
Table 1 Comparison between conventional imaging technology and optical imaging

\begin{tabular}{l|l|l}
\hline Type of imaging & Advantages & Disadvantages \\
\hline $\begin{array}{l}\text { Conventional } \\
\text { imaging } \\
\text { technology (CT/ }\end{array}$ & $\begin{array}{l}\text { (1) Can image exact size of lesions } \\
\text { macroscopically }\end{array}$ & $\begin{array}{l}\text { (1) Does not provide information } \\
\text { on biological features of lesions }\end{array}$ \\
\cline { 2 - 3 } & (2) Can image whole body & $\begin{array}{l}\text { (2) Does not show exact locations } \\
\text { during operation }\end{array}$ \\
\cline { 2 - 3 } & $\begin{array}{l}\text { (3) Enables preoperative diagnosis } \\
\text { and clinical staging }\end{array}$ & $\begin{array}{l}\text { (3) High cost and space } \\
\text { occupying }\end{array}$ \\
\hline $\begin{array}{l}\text { Optical imaging } \\
\text { (fluorescence/ } \\
\text { bioluminescence) }\end{array}$ & $\begin{array}{l}\text { (1) Can image molecules/cells of } \\
\text { interest; enables biological imaging } \\
\text { of lesions }\end{array}$ & $\begin{array}{l}\text { (1) Not good at examining deep } \\
\text { lesions }\end{array}$ \\
\cline { 2 - 3 } & $\begin{array}{l}\text { (2) Low cost and space saving; } \\
\text { enables intra-operative real-time } \\
\text { imaging }\end{array}$ & $\begin{array}{l}\text { (2) Needs fluorescent/ } \\
\text { bioluminescent reagents for } \\
\text { imaging }\end{array}$ \\
\cline { 2 - 3 } & \begin{tabular}{l} 
(3) Relative estimation \\
\hline
\end{tabular}
\end{tabular}

do not show the biological characteristics of the lesion, nor its exact location during the operation. In addition, they require a lot of space and much expense for installation.

On the other hand, optical imaging by fluorescence or bioluminescence will provide biological information with sophisticated probes at low cost and in a small space. Disadvantages of optical imaging are that it requires a customized probe (fluorescence or bioluminescence) and has difficulty in imaging deeper lesions. In addition, signals from optical probes are relative, not absolute (Table 1).

\section{An Optical Probe for In Vivo Imaging of Caspase-3 Activity}

Many optical probes have been developed to visualize protease activities for biological in vivo imaging [1-7]. They have been used to analyze the dynamic changes in the cell and organ stresses and injuries, including hypoxia and reoxygenation $(\mathrm{H} / \mathrm{R})$ and ischemia and reperfusion (I/R) of cells and organs, respectively. $\mathrm{I} / \mathrm{R}$-induced injury is an important concern in various clinical situations, including surgical resection of organs, transplantation of organs and cells, and myocardial/ cerebral/intestinal infarction. In these clinical situations, prolonged ischemia followed by reperfusion results in extended organ injury caused by apoptosis, necrosis, and organ failure [8]. Although the mechanisms of I/R-induced injury are complex, post-ischemic apoptotic and/or necrotic injury play a pivotal role in subsequent organ failure $[9,10]$. Therefore, noninvasive monitoring of cell and organ injury (e.g., caspase activity) in vivo is definitely beneficial and is able to provide important clues for treatment.

We previously developed a novel probe of cyclic luciferase reflecting caspase-3 activity (pcFluc-DEVD) [2]. Two fragments of DnaE inteins are fused with neighboring $\mathrm{N}$-terminal and $\mathrm{C}$-terminal ends of firefly luciferase, which are connected 
with DEVD (Asp-Glu-Val-Asp [a substrate sequence of caspase-3]). After translation into a single polypeptide, the $\mathrm{N}$-terminal and $\mathrm{C}$-terminal ends of the luciferase are ligated by protein splicing, producing a closed circular polypeptide chain. The cyclic luciferase structure is distorted and therefore almost loses its bioluminescent activity. When caspase- 3 is activated in cells, the DEVD chain is cleaved, which restores firefly luciferase (Fluc) luminescent activity by returning to the original structure (Fig. 1) [11].

This cyclic luciferase was first applied for quantitative measurement of caspase-3 activity in living cells. HeLa cells transfected with this probe were stimulated with staurosporine, an inducer of apoptosis. This bioluminescent probe based on cyclic luciferase allowed precise and quantitative measurements of caspase- 3 activity in living cells. Moreover, the response of this cyclic luciferase to caspase- 3 activation was rapid and paralleled biochemically assessed apoptosis.

This imaging technology by cyclic luciferase could be applied for in vivo realtime imaging of caspase- 3 activity in living mice. We successfully imaged the caspase-3 activity in post-ischemic liver using our caspase-3 probe (Fig. 2). By transfecting the adenovirus vector encoding pcFluc-DEVD (AdpcFluc), we investigated whether or not this probe could monitor caspase-3 dependent apoptosis in the mouse liver during I/R (Fig. 2) [11]. We confirmed the efficacy of the probe in detecting apoptotic liver injury in a mouse model, which showed the potency for future clinical application.

\section{bioluminescent probe for Caspase-3 activity -distorted circular luciferase senses caspase-3 activity -}

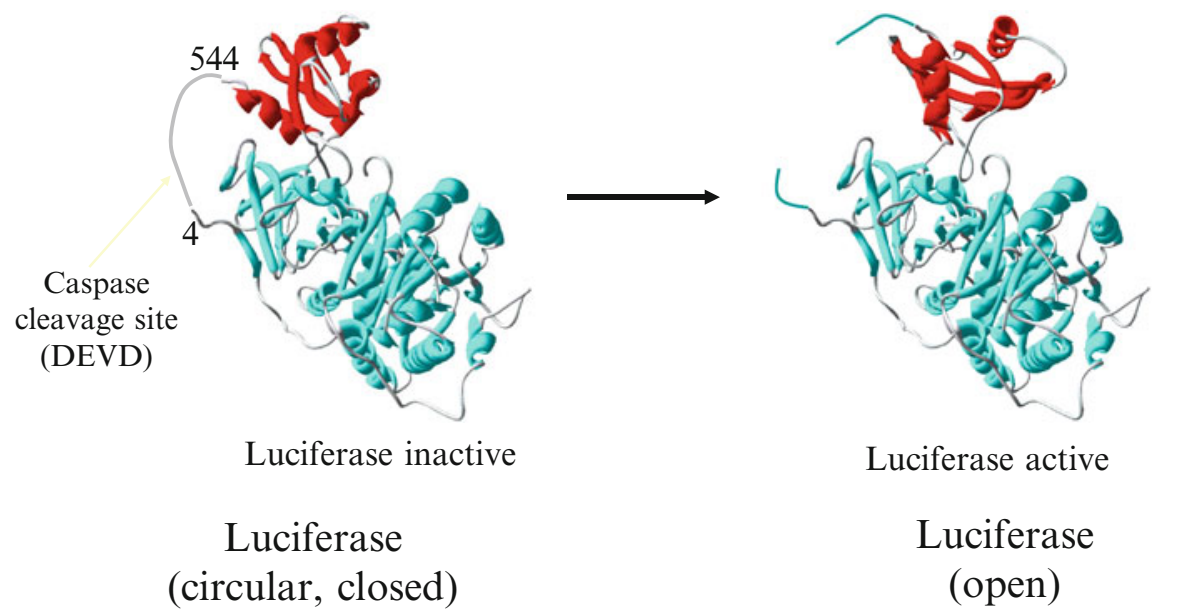

Fig. 1 Bioluminescent probe for caspase-3 activity. The principle for monitoring activity of caspase-3 is based on cyclic firefly luciferase (Modified from Ozaki et al. [11], with permission) 


\section{In vivo imaging of caspase-3 activity by the optic probe}
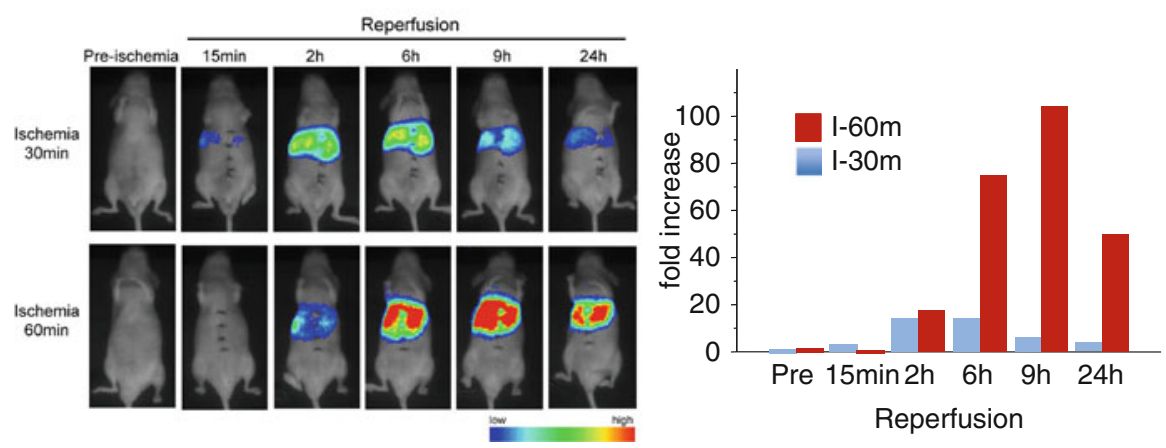

Fig. 2 In vivo imaging of caspase-3 activity by the optical probe. An ischemic insult to the liver induced caspase-3 activation (Modified from Ozaki et al. [11], with permission)

\section{An Optical Probe for In Vivo Imaging of the Hepatic Redox State}

Reactive oxygen species (ROS) generated during I/R of organs certainly play a pivotal role in I/R-induced organ injury and failure [12]. Hepatic I/R results in massive cell death (a) through apoptosis and necrosis mediated by excessive generation of ROS in hepatocytes early after I/R [13]; and (b) through necrosis caused by neutrophilic infiltration in the later phase [14]. According to the numerous studies on liver $\mathrm{I} / \mathrm{R}$ in mice, organ injury in the early post-I/R period is definitely mediated by generation of ROS in hepatocytes $[15,16]$. Excessive ROS directly exert deleterious effects on cells and tissues through lipid peroxidation, protein degradation, and DNA damage. In addition, redox-sensitive molecules, such as nuclear factor-kappa $\mathrm{B}(\mathrm{NF}-\kappa \mathrm{B})$, activator protein 1 (AP-1), and some mitogen-activated protein kinases (MAPKs), are potentially activated under these oxidative conditions and play pivotal roles in I/R-induced injury [17]. Therefore, monitoring cellular redox states by visualizing cellular ROS in post-I/R tissue has been one of the possible strategies for forecasting I/R-induced injury and failure [18]. These considerations suggest that the post-I/R redox state may be a good prognostic indicator of liver injury and function after liver surgery and transplantation, implying that in vivo monitoring may provide more diagnostic and therapeutic options in clinical liver surgery and transplantation.

For in vivo assays of tissue oxidative stress (OS), tissues are usually homogenized and subsequently assayed, either with redox-sensing electrodes or by measuring the ratios of the reduced and oxidized forms of glutathione and ascorbate. Recently, the redox states of tissues have also become assessable by using the dyes 5- (and 6-) carboxy-2,7-dichlorodihydrofluorescein diacetate [19] and dihydrofluorescein diace- 


\section{In vivo imaging of I/R-induced oxidative stress of mouse liver}

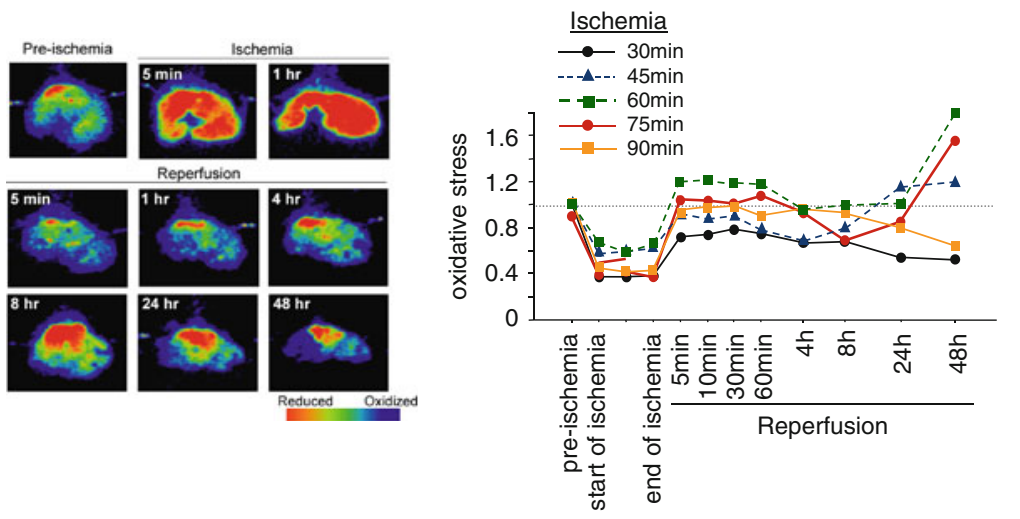

Fig. 3 In vivo imaging of I/R-induced oxidative stress of the mouse liver. The roGFP probe showed dynamic changes in liver redox states. The left panel depicts representative images of the dynamic changes after $60 \mathrm{~min}$ of ischemia, followed by reperfusion (oxidation, green to blue; reduction, orange to red) (Modified from Haga et al. [10], with permission)

tate [20]. Although such approaches allow evaluation of the sum of the oxidized and reduced species present, and thereby estimation of the overall redox state of the tissue, these techniques do not allow measurement of redox potentials in real time. More importantly, homogenization of tissues precludes the possibility of monitoring dynamic changes in OS in the same animal and organ.

A newly developed reduction-oxidation-sensitive green fluorescent protein (roGFP) probe (GFP with mutations of C48S, S147C, Q204C, and S65T) [1] could be applied for in vivo imaging. Use of the roGFP probe enables evaluation of cell and organ redox states non-invasively and continuously. Adenovirally transduced roGFP in the mouse liver was shown to act as a good indicator of pre- and postischemic liver redox states (Fig. 3) [10]. Through use of this roGFP probe, postoperative liver injury may be predicted by real-time monitoring of liver redox states.

\section{Optical Imaging of Deeper Lesions in the Living Body}

An increasing number of antibodies have been applied for targeting antigens on the surface of cancer cells for clinical diagnosis and therapy, on the basis of the fact that some specific antigens expressed on the cancer cell surface reflect malignant behaviors including invasion, metastasis, and neo-vascularization [21-23]. Realtime visualization of tumor-specific antigens in the living body will enable timely understanding of cancer expansion and its biological behaviors, and prediction of the patient's prognosis in the near future. 
Fluorescence and bioluminescence imaging technologies have played important roles in molecular imaging in cells and small animals [24-26]. Photon detection is easy to use, cost saving, and space saving, compared with radioisotope imaging. Basically, bioluminescence imaging is achieved with a luciferin-luciferase reaction in the presence of sufficient amounts of molecular oxygen and adenosine triphosphate (ATP). However, most bioluminescence spectra are observed in the visible region, which overlaps with the absorption spectra of hemoglobin and tissue constituents, therefore attenuating the bioluminescence intensity in living animals.

Recently, a self-illuminating quantum dot probe was developed to improve the light penetration based on bioluminescence resonance energy transfer (BRET) between the bioluminescence of Renilla luciferase and quantum dots [27]. The multivalent conjugation of Renilla luciferase to single dots allowed for highly efficient BRET between luciferase and quantum dots. However, the large size of the conjugate caused problems in metabolism and localization in vivo [28]. Several far-red fluorescent protein variants showing maximal emission at around $650 \mathrm{~nm}$ have been developed for in vivo imaging [29], but are not well characterized as energy acceptors for BRET systems. On the other hand, the organic dye indocyanine and its derivatives have molecular weights of less than 1,200 Da; they produce far-red fluorescence and have been widely used for in vivo imaging [30].

Luciferase conjugated to such organic dyes is expected to create new possibilities for in vivo application. Cypridina luciferase (CLuc) catalyzes the oxidation of Cypridina luciferin to yield light emission peaking at $460 \mathrm{~nm}$ [31].

The luciferase genes from both Cypridina hilgendorfii and C. noctiluca have been cloned [32], the latter of which we used. This self-secretory CLuc of $62 \mathrm{kDa}$ has some unique properties as a bioluminescent enzyme [33]. The secreted protein contains 17 disulfide bond pairs and is highly stable under physiological conditions. Its turnover rate (1400 luciferin molecules per minute) is the highest among known luciferases [34]. We have expressed recombinant CLuc in yeast and applied it to ELISA. We conjugated a far-red fluorescent indocyanine derivative to biotinylated CLuc via glycol chains and named this far-red bioluminescent protein (FBP).

A monoclonal antibody against human Delta-like protein (anti-Dlk1), one of the embryonic antigens expressed on the surface of many cancer cells, including hepatocellular carcinoma, was then produced. Using anti-Dlk1 monoclonal antibody linked to FBP via biotin-avidin interaction, we achieved bioluminescence imaging of cancer cells in vivo in mice (Fig. 4).

However, very unfortunately, there were some non-specific signals from the tissues that did not express Dlk-1, though the tumor implanted into the liver itself was imaged clearly by this probe. This may have been because excessive and unbound antibodies circulating in the blood or captured in tissues emitted signals when the substrate was injected. In order to apply this probe to clinical application, nonspecific signals must be reduced as much as possible. So, we have launched the following new project. 
In vivo imaging by a new optic probe for deeper lesions in mouse
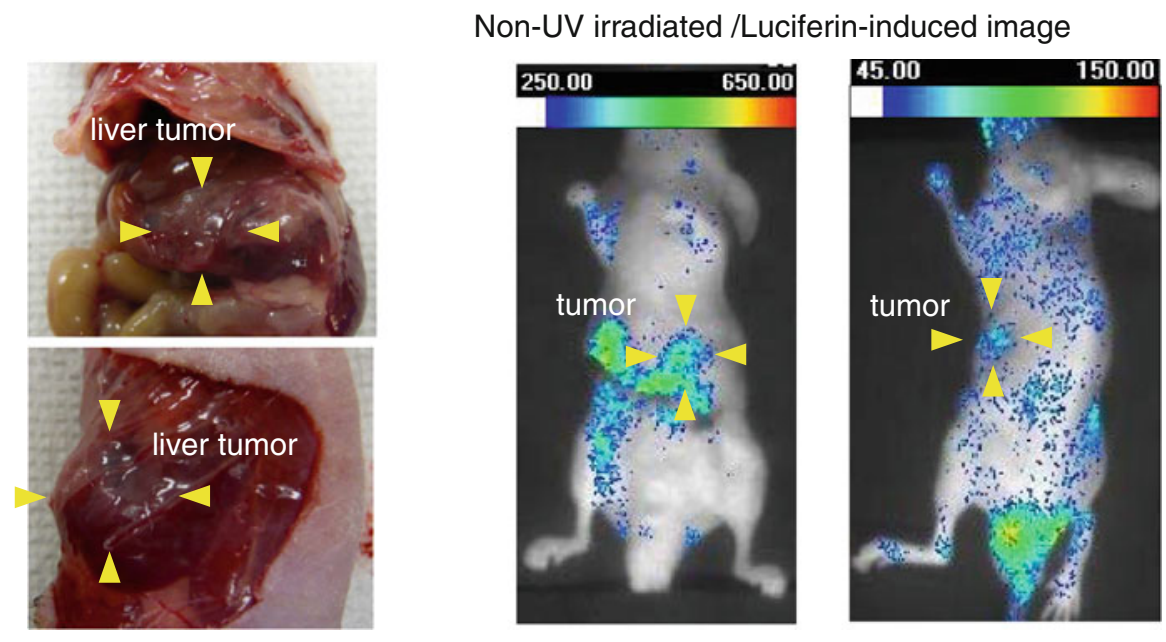

Image was obtained $24 \mathrm{hr}$ after Luciferin adm (iv)

(400mg/200ml i.v.)

Fig. 4 In vivo imaging by a new optical probe for deeper lesions in the mouse. A liver tumor of the mouse was imaged, but some non-specific signals were observed, probably because of excessive and unbound antibodies

\section{Development of an In Vivo Optical Probe for Antigen-Specific Imaging Inside/Outside Cells}

For accurate biological diagnosis and rational therapy in clinical practice, we need to know the biological features of the lesion as well as its size, shape, and extent. For this purpose, we plan to develop a new optical probe, which will image the target molecules only after binding to the antigen. This does not allow unbound antibody to emit light signals, and therefore it will lead to the reduction of nonspecific signals in vivo. Additionally, the probe should be applied to targets inside as well as outside cells in vivo, because there are lots of diagnostic and therapeutic targets inside as well as outside cells. In order to create a new type of in vivo optical probe, the following two technologies have to be developed (Fig. 5).

(A) Development of an Optical Probe Activated Only When it Binds to the Target Molecule

In order to develop a probe activated after binding to the target antigen in vivo, we have designed a new type of luciferase-antibody conjugated probe. We planned to prepare split firefly luciferases, the $\mathrm{N}$-terminal and C-terminal fragments of luciferase conjugated with SNAP (a 182-residue polypeptide that can be fused to any 


\section{Strategy for Ag-specific imagingin vivo}

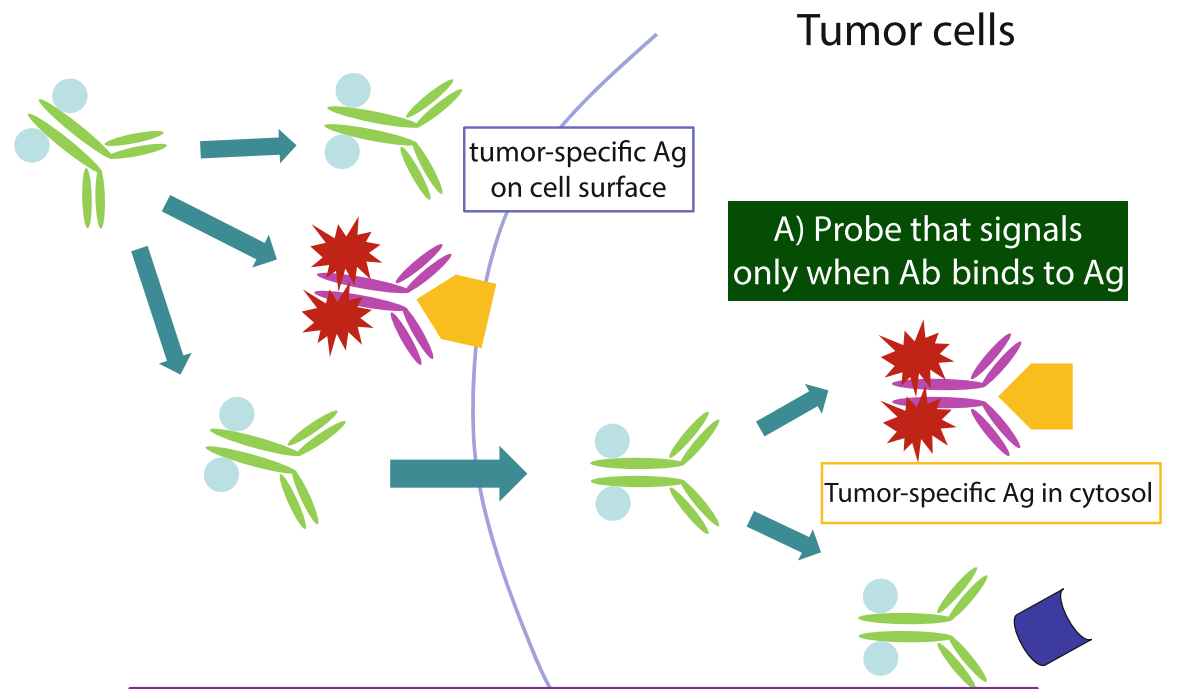

B) Safe and stable delivery of a probe into cell/cytosol

Fig. 5 Strategy for antigen-specific imaging in vivo

protein of interest) - FlucN-SNAP and FlucC-SNAP, respectively - and to prepare a polyclonal antibody covalently connected with benzylguanine (Ab-BG). When the antibodies with FlucN and FlucC recognize and bind to the close epitopes of the same antigen, split luciferase fragments will be reconstituted and activated (Fig. 6). Theoretically, the probe should enable us to see the target molecule in tissues and cells only when it exists.

We plan first to express FlucN-SNAP and FlucC-SNAP proteins by Escherichia coli, and then to bind benzylguanine (BG) with polyclonal Ab. Lastly, we will conjugate these proteins (Fig. 7).

To date, we have succeeded in obtaining sufficient amounts of FlucN-SNAP and FlucC-SNAP proteins by the E. coli expression system, and we have confirmed the good binding capacity of FlucN-SNAP and FlucC-SNAP proteins to BG. So, we have bound $\mathrm{BG}$ with polyclonal $\mathrm{Ab}$, and performed conjugation with Fluc-SNAPs. We are now examining the probe function in our in vitro system.

(B) Development of a Safe and Stable System for Delivery of Optical Probes into Cells

In order to develop a new system to deliver sufficient amounts of optical probe safely into cells in vivo, we are now developing a new liposome-based delivery system.

There seem to be two important processes for developing an effective and rapid delivery system (Fig. 8). First, the carrier including probes in it should be 


\section{Concept for antigen-dependent optic probe}

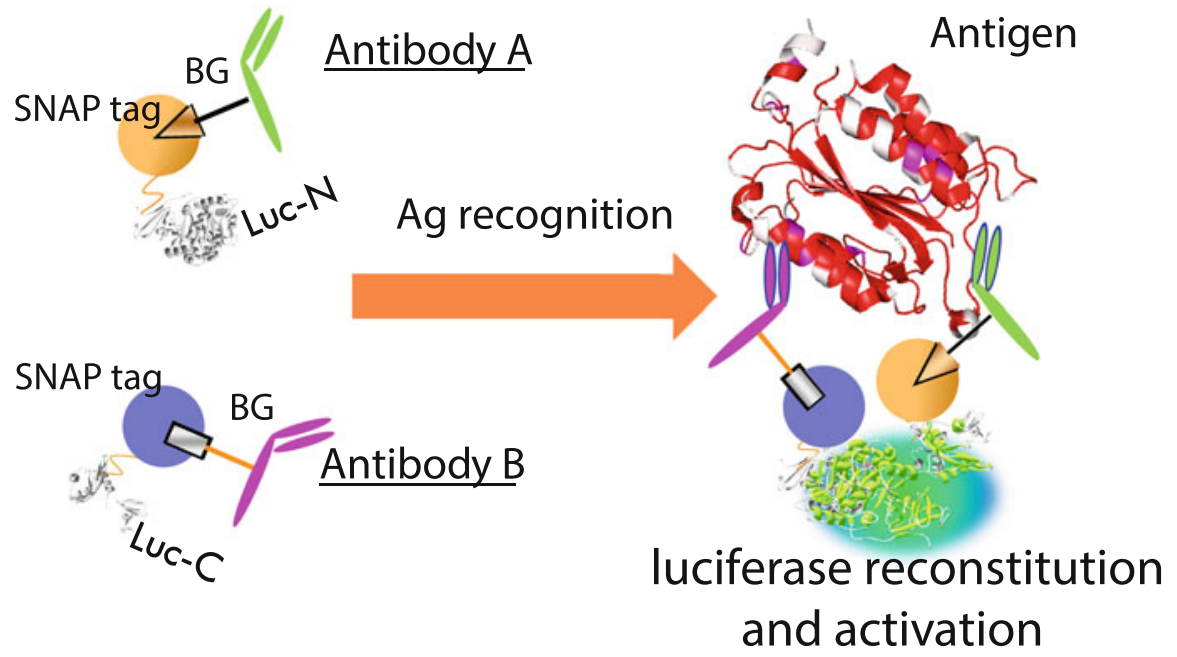

Fig. 6 Concept for an antigen-dependent optical probe. When the probes with C-/N-terminal fragments of luciferase recognize the antigen, luciferase is reconstructed and activated

\section{Procedures of making a probe}

\section{(1) Expression of FlucN-SNAP \& FlucC-SNAP}

\begin{tabular}{|c|c|c|}
\hline FlucN & SNAP & $6 \mathrm{His}$ \\
\hline FlucC & SNAP & $6 \mathrm{His}$ \\
\hline
\end{tabular}

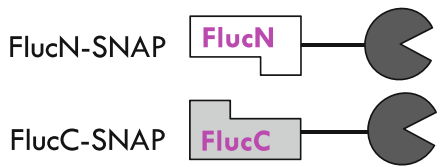

(2) Binding BG (Ligand) to polyclonal $\mathbf{A b}$

$\checkmark$ :Benzylguanine group

SNAP-tagged
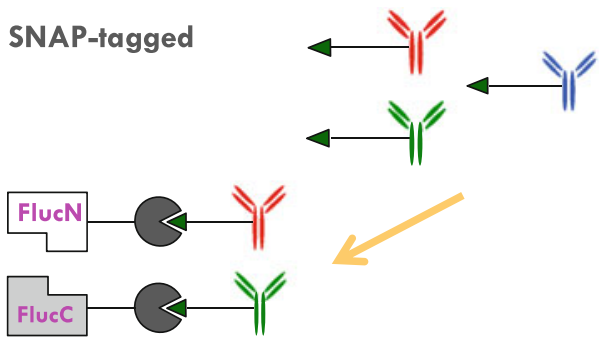

(3) Conjugation of Fluc fragments and antibodies by SNAP-BG binding

Fig. 7 Procedure for making a probe: (1) expression of FlucN-SNAP and FlucC-SNAP; (2) binding BG (ligand) to polyclonal Ab; (3) conjugation of Fluc fragments and antibodies by SNAP-BG binding 


\section{Strategy for cellular delivery of the probe}

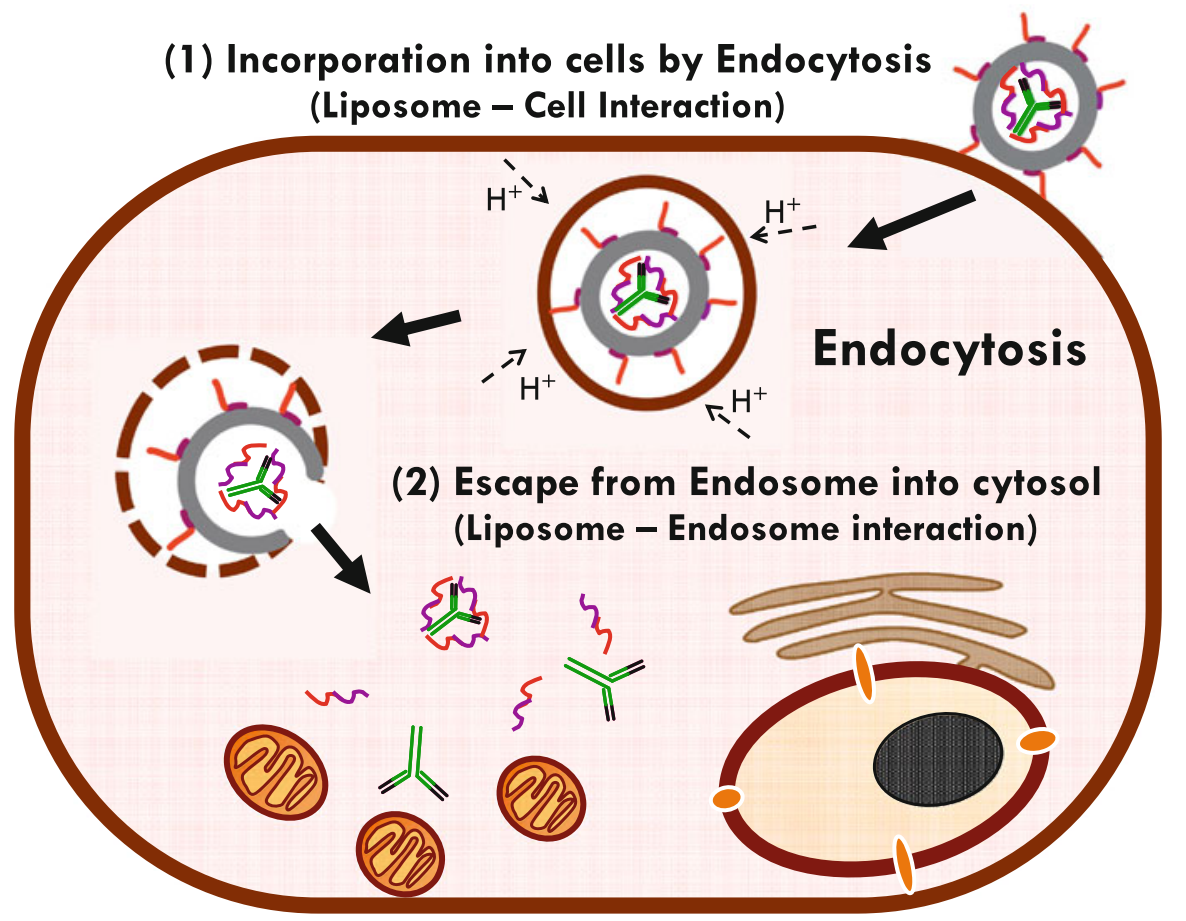

Fig. 8 Two important processes for efficient cellular delivery of probes: (1)incorporation into cells by endocytosis (a liposome-cell interaction); (2) escape from the endosome into the cytosol (a liposome-endosome interaction)

incorporated into cells sufficiently and rapidly. Second, the incorporated probe should be released from the lysosome into the cytosol. These processes should not be harmful to the cells and the probes.

We have already made a prototype liposome-based carrier for efficient drug delivery in vivo. We further added a cell-permeable octa-arginine peptide (R8) to the liposome, expecting better incorporation into cells (Fig. 9). In the in vitro experiments, the R8 liposome successfully delivered the "antibody" into the cells much better than the liposome without R8. The R8 liposome, however, failed to escape the "antibody" from the endosome into the cytosol.

Next, we applied GALA to make the probe release rapidly and efficiently from the endosome into the cytosol (Fig. 10). GALA is a 30-amino-acid peptide with a glutamic acid-alanine-leucine-alanine repeat that contains a histidine and tryptophan residue. GALA changes its conformation from a random coil to an amphipathic alpha-helix when $\mathrm{pH}$ values are between 7.0 and 5.0. At neutral $\mathrm{pH}$, GALA is water soluble, while at acidic $\mathrm{pH}$, GALA binds to bilayer membranes. So it is expected to promote rapid fusion of the liposome with the endosomal membrane in the cell. The fusogenic liposome was modified with a cell-penetrating R8 and also GALA. 
Multi-functional Envelope-type Nano Device (MEND) : R8-Liposome

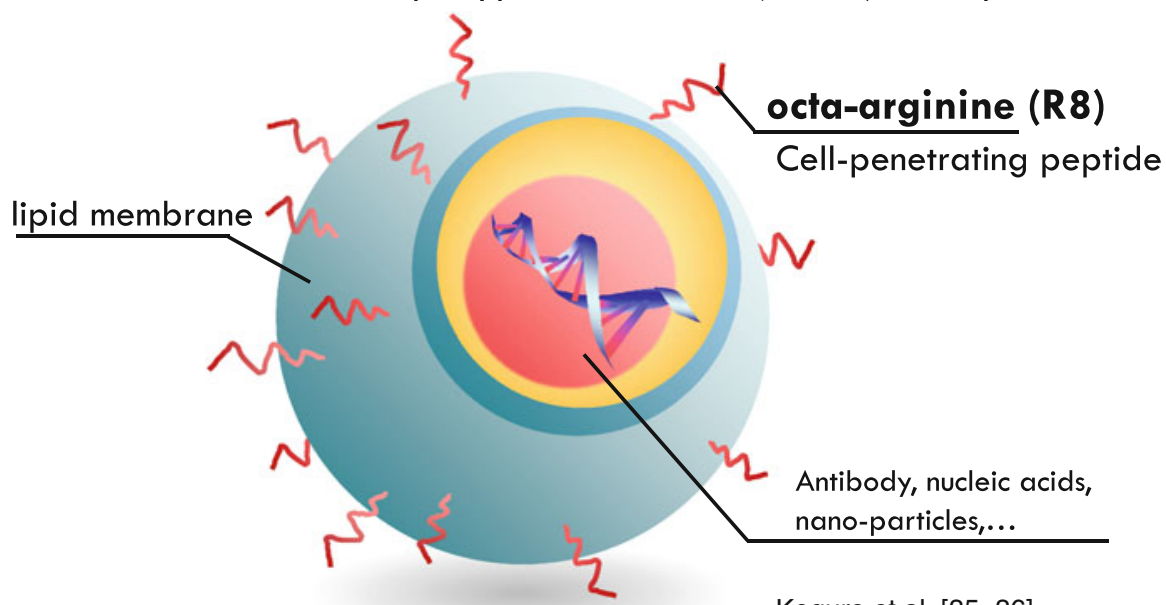

Kogure et al. [35, 36]

Nakamura et al. [37]

Fig. 9 Concept of a multifunctional envelope-type nano device (MEND). Octa-arginine (R8) is bound to the liposome surface (R8 liposome)

\section{GALA: $\mathrm{pH}$ sensitive fusogenic peptide}

\section{GALA: WEAALAEALAEALAEHLAEALAEALEALAA}
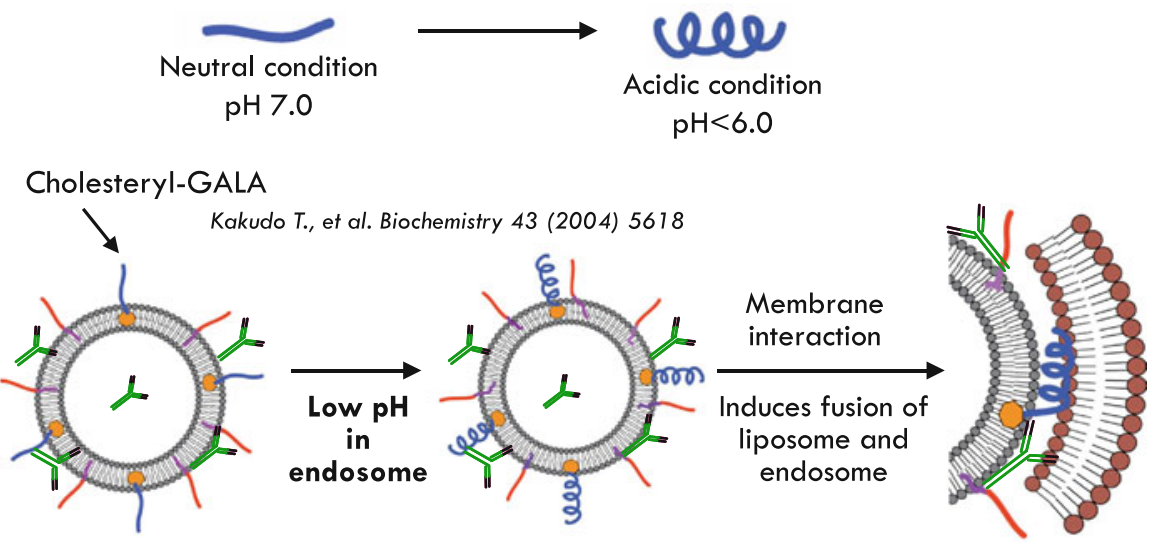

Fig. 10 The GALA pH-sensitive fusogenic peptide changes its conformation depending on $\mathrm{pH}$ values and is expected to promote lipid-membrane fusion 
The presence of R8 enhanced the cellular uptake of antibodies, while the mounting of GALA reduced the endosomal entrapment, resulting in release of the antibody into the cytosol within $30 \mathrm{~min}$ in in vitro experiments. Moreover, compared with the commercially available reagents for protein delivery, the R8/GALAmodified liposome was superior in terms of both cellular uptake and transport into the cytosol. The specific antibody delivered by the R8/GALA liposome was detected by its epitope, which indicated that the R8/GALA liposome basically did not deteriorate the protein function.

\section{Conclusion}

In this chapter, we have described possible application of an optical probe for "biological imaging" and our recent challenge in undertaking development of a new type of in vivo probe.

In the mouse liver I/R model, the fluorescent probe, roGFP, and bioluminescent luciferase probe, pcFluc-DEVD, were useful in understanding the dynamic changes of liver redox states and apoptotic cell death. We could understand the pathological significance of OS and apoptosis in I/R-induced liver injury. Because of the difficulty of imaging of deeper lesions by optical probes, we developed a unique probe loaded on an antibody. This probe successfully imaged a target molecule in deeper regions in mice, but also revealed another problem - an in vivo non-specific signal from excessive and unbound antibodies. Lastly, we undertook the challenge to develop an innovative optical probe which switches "on" only when the probe recognizes a target molecule. The project of developing this unique probe is still underway but seems very promising at this time.

Regarding a carrying system of the probe to target molecules in vivo, we developed a liposome bound with a cell-penetrating R8 and GALA. The R8/GALA liposome delivered the "antibody" to the cells and also the cytosol efficiently and rapidly in vivo.

In the future, we plan to prove the efficacy of the new probe carried by the R8/ GALA liposome in in vivo experiments.

Open Access This chapter is distributed under the terms of the Creative Commons Attribution Noncommercial License, which permits any noncommercial use, distribution, and reproduction in any medium, provided the original author(s) and source are credited.

\section{References}

1. Dooley CT, Dore TM, Hanson GT et al (2004) Imaging dynamic redox changes in mammalian cells with green fluorescent protein indicators. J Biol Chem 279:22284-22293

2. Kanno A, Yamanaka Y, Hirano H et al (2007) Cyclic luciferase for real-time sensing of caspase-3 activities in living mammals. Angew Chem Int Ed 46:7595-7599

3. Zhu L, Xie J, Swierczewska M et al (2011) Real-time video imaging of protease expression in vivo. Theranostics 1:18-27 
4. Lexman B, Hall DE, Bhojani MS et al (2002) Noninvasive real-time imaging of apoptosis. Proc Natl Acad Sci U S A 99:16551-16555

5. Ray P, De A, Patel M et al (2008) Monitoring caspase-3 activation with a multimodality imaging sensor in living subjects. Clin Cancer Res 14:5801-5809

6. Yhee JY, Kim SA, Koo H et al (2012) Optical imaging of cancer-related proteases using nearinfrared fluorescence matrix metalloproteinase-sensitive and cathepsin B-sensitive probes. Theranostics 2:e0017

7. Kim GB, Kim Y-P (2012) Analysis of protease activity using quantum dots. Theranostics 2:e004

8. Ozaki M, Deshpande SS, Angkeow P et al (2000) Inhibition of the Rac1 GTPase protects against nonlethal ischemia/reperfusion-induced necrosis and apoptosis in vivo. FASEB $J$ $14: 418-429$

9. Lucchesi BR (1993) Complement activation, neutrophils, and oxygen radicals in reperfusion injury. Stroke 24:I41-I47

10. Haga S, Remington S, Morita N et al (2009) Hepatic ischemia induced immediate oxidative stress after reperfusion and determined the severity of the reperfusion-induced damage. Antioxid Redox Signal 11(10):2563-2572

11. Ozaki M, Haga S, Ozawa T (2012) In vivo monitoring of liver damage by caspase-3 probe. Theranostics 2(2):207-214

12. Robin E, Guzy RD, Loor G et al (2007) Oxidant stress during simulated ischemia primes cardiomyocytes for cell death during reperfusion. J Biol Chem 282:19133-19143

13. Tsung A, Kaizu T, Nakao A et al (2005) Ethyl pyruvate ameliorates liver ischemia-reperfusion injury by decreasing hepatic necrosis and apoptosis. Transplantation 79:196-204

14. Montalvo-Jave EE, Escalante-Tattersfield T, Ortega-Salgado JA et al (2008) Factors in the pathophysiology of the liver ischemia-reperfusion injury. J Surg Res 147:153-159

15. Haga S, Terui K, Fukai M et al (2008) Preventing hypoxia/reoxygenation damage to hepatocytes by p66(shc) ablation: up-regulation of anti-oxidant and anti-apoptotic proteins. J Hepatol 48:422-432

16. Terui K, Enosawa S, Haga $S$ et al (2004) Stat3 confers resistance against hypoxia/ reoxygenation-induced oxidative injury in hepatocytes through upregulation of Mn-SOD. J Hepatol 41:957-965

17. Thannickal VJ, Fanburg BL (2000) Reactive oxygen species in cell signaling. Am J Physiol Lung Cell Mol Physiol 279:L1005-L1028

18. Guo L, Haga S, Enosawa $S$ et al (2004) Improved hepatic regeneration with reduced injury by redox factor-1 in a rat small-sized liver transplant model. Am J Transplant 4:879-887

19. Jiang J, Kini V, Belikova $\mathrm{N}$ et al (2004) Cytochrome $\mathrm{c}$ release is required for phosphatidylserine peroxidation during Fas-triggered apoptosis in lung epithelial A549 cells. Lipids 39:1133-1142

20. Jones MA, Raymond MJ, Yang Z et al (2007) NADPH oxidase-dependent reactive oxygen species formation required for root hair growth depends on ROP GTPase. J Exp Bot $58: 126-127$

21. Adams R, Meade A, Wasan H et al (2008) Cetuximab therapy in first-line metastatic colorectal cancer and intermittent palliative chemotherapy: review of the COIN trial. Expert Rev Anticancer Ther 8:1237-1245

22. Boltze J, Förschler A, Nitzsche B et al (2008) Permanent middle cerebral artery occlusion in sheep: a novel large animal model of focal cerebral ischemia. J Cereb Blood Flow Metab 28:1951-1964

23. Ilowite NT (2008) Update on biologics in juvenile idiopathic arthritis. Curr Opin Rheumatol 20:613-618

24. Contag PR, Olomu IN, Stevenson DK et al (1998) Bioluminescent indicators in living mammals. Nat Med 4:245-247

25. Hoffman RM, Yang M (2005) Dual-color, whole-body imaging in mice. Nat Biotechnol 23:790 
26. Ntziachristos V, Ripoll J, Wang LV et al (2005) Looking and listening to light: the evolution of whole-body photonic imaging. Nat Biotechnol 23:313-320

27. So MK, Xu C, Loening AM et al (2006) Self-illuminating quantum dot conjugates for in vivo imaging. Nat Biotechnol 24:339-343

28. Frangioni JV (2006) Self-illuminating quantum dots light the way. Nat Biotechnol $24: 326-328$

29. Shcherbo D, Merzlyak EM, Chepurnykh TV et al (2007) Bright far-red fluorescent protein for whole-body imaging. Nat Methods 4:741-746

30. Frangioni JV (2003) In vivo near-infrared fluorescence imaging. Curr Opin Chem Biol 7:626-634

31. Shimomura O, Johnson FH (1971) Mechanism of the luminescent oxidation of Cypridina luciferin. Biochem Biophys Res Commun 44:340-346

32. Thompson EM, Nagata S, Tsuji FI (1989) Cloning and expression of cDNA for the luciferase from the marine ostracod Vargula hilgendorfii. Proc Natl Acad Sci U S A 86:6567-6571

33. Shimomura O, Johnson FH, Saiga Y (1961) Purification and properties of Cypridina luciferase. J Cell Comp Physiol 58:113-123

34. Shimomura O, Johnson FH, Masugi T (1969) Cypridina bioluminescence: light-emitting oxyluciferin-luciferase complex. Science 164:1299-1300 